\title{
LAS CUESTIONES SOCIOCIENTÍFICAS: UNA ALTERNATIVA DE EDUCACIÓN PARA LA SOSTENIBILIDAD
}

\author{
NIDIA YANETH TORRES MERCHÁN ${ }^{1}$ \\ yanethtorres3@hotmail.com
}

Manizales, 2010-09-14 (Rev. 2011-04-24)

\section{RESUMEN}

El artículo presenta una reflexión acerca de la utilización y abordaje de las cuestiones sociocientíficas (CS) en futuros docentes. Esta estrategia didáctica genera inquietudes relacionadas con la influencia de la ciencia y la tecnología en la sociedad y en el ambiente (CTSA), promueve compromisos en la formación de actitudes y comportamientos favorables hacia el logro de un desarrollo sostenible, y reflexiona acerca de los diversos problemas ambientales, producto de intereses individuales alejados de la conservación biológica y cultural. Las CS permiten vincular de manera más llamativa diversos temas disciplinares, no solamente con el fin de obtener un aprendizaje cognitivo en los estudiantes, sino también el desarrollo de capacidades sociales, éticas, culturales que promueven una educación para la sostenibilidad con acciones encaminadas a actuar con responsabilidad social en la toma de decisiones fundamentadas; por ello, se abordaran en el escrito, las nociones acerca del enfoque CTSA, las ventajas de la utilización de las CS, ilustrando finalmente las reflexiones que requieren hacer los docentes.

PALABRAS CLAVE: Cuestiones sociocientíficas, educación para la sostenibilidad, ciudadanía, toma de decisiones.

THE SOCIAL-SCIENTIFIC MATTERS: AN EDUCATION ALTERNATIVE FOR SUSTAINABILITY

\section{ABSTRACT}

The article presents a reflection on the use and dealing with the social-scientific matters (SC) in future teachers. This teaching strategy generates concerns related with the influence of science and technology in society and in the environment (CTSE), promotes compromises in the education of attitudes and behaviors which are favorable for the achievement of a sustainable development, and reflects about the diverse environmental problems product of individual interests away from biological and cultural conservation. The SC allow the connection in a more striking way with a diversity of disciplinary topics, not only in order to obtain a cognitive learning in the students, but also the development of social, ethics and cultural capacities which promote an education for the sustainability with actions channeled to responsible social behavior when making decisions with a foundation. For this reason, the notions about the CTSE approach and the advantages of using the CS are dealt with, thus illustrating the reflections that teachers are requested to make.

Key words: social-scientific matters, education for sustainability, citizenship, decision-making.

\section{INTRODUCCIÓN}

Uno de los principales compromisos de los docentes en la formulación de propuestas curriculares, deber ser la inclusión de situaciones que apoyen la formación de ciudadanos para actuar con responsabilidad social; personas capaces de tomar decisiones que no siempre busquen un beneficio individual, sino también un beneficio colectivo (McComas y Olson, 1998), y además promuevan estrategias que evalúen las bases que acompañan el proceso de formación.

Desde la enseñanza de las Ciencias Naturales se han abordado las cuestiones sociocientíficas (CS), que no solo permiten enseñar los contenidos propios de las ciencias, sino que considera aspectos que habitualmente no son tenidos en cuenta como la tecnología, el ambiente, la sociedad, la educación en valores, la cultura, entre otros (Acevedo, 1995).

Las CS son asuntos tecnocientíficos controvertidos, actuales y de interés social, que permiten la discusión de temas como el calentamiento global, la utilización de antibióticos para la producción animal, la clonación, la experimentación en animales, las fumigaciones aéreas, la utilización de teléfonos móviles, entre otros. Estas situaciones provocan diversas reacciones en los alumnos que les permiten promover una educación dialógica, donde se debaten las hipótesis y supuestos 
esenciales a una investigación científica, así como las implicaciones que esas hipótesis y supuestos tienen para los resultados que pueden producir tales investigaciones; promueve discusiones de las diferentes formas en que podrían diseñarse las investigaciones, y comprende la ciencia como actividad humana influenciada por la cultura, la política y la sociedad.

Diversos problemas globales como: el agotamiento de recursos fundamentales, la degradación de todos los ecosistemas, la pérdida de diversidad biológica y cultural, la pobreza extrema de miles de millones de seres humanos, la amenaza de un cambio climático, la drogadicción, la acumulación de residuos sólidos, la guerra química, los pesticidas en los alimentos, la experimentación con modelos animales, los alimentos transgénicos, la síntesis de xenobióticos, requieren urgente que las instituciones educativas promuevan cambios de actitudes y comportamientos en los estudiantes, que les permitan asumir posiciones sólidas respecto a problemáticas ambientales y sociales, y hacer frente a los problemas socioambientales que afectan a la humanidad (Rubba y Harkness, 1993; Rubba, Schoneweg-Bradford y Harkness, 1996; Lederman, 1999; Acevedo, 2006).

Consientes de que los problemas globales a los que se enfrenta la humanidad, requieren urgente la atención por parte de los educadores, es hora de comprender que las acciones humanas tienen consecuencias que no se pueden desconocer. La educación no puede continuar con la acumulación de conocimientos socialmente descontextualizados, ignorando la crisis ambiental que se presenta. Es entonces necesario empezar a aceptar los límites de capacidad de carga de la tierra, comprender la urgente necesidad de formación en valores como: la solidaridad, la cooperación, la comprensión, superando los valores dominantes como la competencia y el consumismo. ColucciGray, L., Camino, E., Barbiero, G., y Gray, D. (2006), hacen referencia a un sistema de valores que ha surgido encaminado a promover la sensibilidad hacia la tierra, y los relacionan con una "ética del planeta". Así, se hace fundamental crear una conciencia social que cambie la tradición de indiferencia hacia el ambiente.

Las CS permiten la integración de temas ambientales al currículo, y hacen posible la transformación completa de un tema tradicional ya existente, en una propuesta problémica que requiere reflexión, pensamiento crítico y toma de decisiones; posibilitan reflexiones profundas en torno al compromiso que deben adquirir los docentes formadores de formadores y los futuros docentes, respecto a la contribución de una educación con acciones de ciudadanía.

A partir de lo anterior, se presentan en este escrito las generalidades acerca del enfoque CTSA y las CS en procesos de enseñanza, se muestran las reflexiones que realizan los futuros docentes al abordar de manera particular una cuestión sociocientífica, de acuerdo a las problemáticas que podrían afectar su práctica profesional y personal.

\section{METODOLOGÍA}

En este trabajo participaron 15 estudiantes que cursan estudios en programas de la Facultad de Educación, en la Licenciatura de Psicopedagogía y en Ciencias Naturales y Educación Ambiental de la Universidad Pedagógica y Tecnológica de Colombia (UPTC). La edad de los participantes está entre los 17 y los 20 años.

Con el grupo de estudiantes se realizaron sesiones de discusión del enfoque CTSA con la utilización de CS, a partir de un seminario que destaca la necesidad de asumir una posición crítica y sólida, encaminada a generar actitudes que permitan reflexionar no solo la profesión del docente, sino la responsabilidad de prepararse para abordar diversas situaciones controversiales; en la electiva Socio-humanística denominada "Cuestiones Sociocientíficas".

\section{RESULTADOS Y DISCUSIÓN}

El seminario fue desarrollado en torno a la reflexión y discusión de diversos artículos científicos. Primero se abordaron las generalidades del enfoque CTSA y las CS, de manera que se destacan las ventajas en la utilización de este enfoque didáctico, y se consideran las habilidades que el docente debe tener y desarrollar para contribuir a una educación para la sostenibilidad.

\section{Educación CTSA facilitadora del abordaje de las CS}


El movimiento CTSA tiene su origen en la crisis ambiental y en las reflexiones sobre la naturaleza de la ciencia y su papel en la sociedad. Con este enfoque de enseñanza, se busca rescatar valores y principios dentro del contexto contemporáneo para referir las perspectivas futuras, visualizando una educación científica transformadora, en una perspectiva reflexiva sobre el modelo del desenvolvimiento tecnológico y sobre el modelo de consumo actual (Aikenhead, 1997).

Este enfoque permite asumir un compromiso para que toda la educación, tanto formal como informal (escuelas, colegios, universidades, museos, medios de comunicación), presten atención a la situación del mundo; de manera que se facilite una percepción correcta de los problemas ambientales, y se fomenten actitudes y comportamientos favorables para el logro de un futuro sostenible (Vilches y Gil-Pérez, 2003). Hace énfasis en la necesidad de que los docentes formadores de docentes y docentes en formación, incorporen en su práctica pedagógica una educación para la sostenibilidad, orientada a la formación de ciudadanos que se interesen por la situación de emergencia planetaria que se presenta. Así, Wang y Schmidt (2001) sugieren la necesidad de promover en el aula de clase un clima de atención y vinculación de problemas controversiales, que comprendan contextos socioculturales, políticos y económicos, donde se asuman con responsabilidad social diversas problemáticas, producto del desarrollo científico y tecnológico. La utilización de CS, forma a los estudiantes para que tengan una participación activa y fundamentada en la sociedad, y se construyan una imagen más real acerca de la acción científica.

En la electiva interdisciplinar denominada "Cuestiones Sociocientíficas", los estudiantes (E) manifiestan con comentarios, como los que se presentan a continuación, la valoración de las CS como alternativa de educación para la sostenibilidad:

E1. El abordaje de las CS permite la participación ciudadana en la toma de decisiones a la hora de consumir productos y lo que estos productos pueden ocasionar al medio ambiente.

E2. Las CS son un componente que fortalece al ser humano en su capacidad de reflexión y crítica; hacen pensar que los recursos no duran para siempre y que debemos desarrollar hábitos de conservación; por lo tanto hay que generar conciencia en todas las generaciones.

E3. Las CS permiten unir los asuntos científicos y tecnológicos con la sociedad, cuestiona lo importante que es dejar ser consumistas.

E4. Esta experiencia me permitió ver que la ciencia no es exacta, que tiene ventajas y desventaja, las CS tienen como finalidad generar controversias dentro de la sociedad creando un espíritu de crítica frente a las problemáticas y temáticas y desarrollo del mundo en el contexto de la ciencia, la tecnología y la sociedad.

El abordaje de las CS requiere la inclusión de aspectos acerca de la naturaleza de la ciencia, la tecnología y su influencia en la sociedad y en el ambiente, de manera que se comprenda que los procesos de enseñanza no solo deben estar centrados en aspectos conceptuales, sino que se deben incorporar en el currículo con una perspectiva crítica aspectos políticos, culturales, axiológicos que promuevan cambios de estilo de vida y una necesidad de reflexionar nuestra capacidad de consumidores para cambiarla (Comín y Font, 1999)

La discusión de las CS permite construir una imagen más real de los avances científicos y tecnológicos, encaminada a promover actitudes de ciudadanía responsable, que se constituye en un elemento esencial para una educación moral ayudando a los alumnos a comprender situaciones sociales. De acuerdo a Reis (2001), estos problemas sociocientíficos son poco delimitados, multidisciplinares, heurísticos, cargados de valores (involucrando valores estéticos, ecológicos, morales, educativos y religiosos), lo cual permite hacer un reflexión integral acerca de los efectos que puedan tener las acciones de cada persona.

El abordaje de las CS juega un papel importante en la formación de profesionales de la educación, que los prepara para participar en controversias sobre temas sociales, políticos, éticos, morales y ambientales, lo cual exige la formación de ciudadanos críticos dotados de conocimientos y capacidades, que les permitan actuar responsablemente en la toma de decisiones fundamentadas en una sociedad democrática, promoviendo siempre un activismo ciudadano en la comunidad, donde se es consciente de que las actitudes de adaptación ante la emergente crisis global no contribuyen y, por el contrario, sí perjudican.

Lo anterior, puede ser evidente en los comentarios que hicieron los estudiantes (E) cuando se les preguntó: ¿Cuál ha sido la contribución de la electiva "Cuestiones Sociocientíficas" en su formación profesional?: 
E1. He aprendido que no debo conformarme con lo que sé; como por ejemplo cuando se consume un medicamento, generalmente uno no se detiene a consultar los componentes de éste y los efectos colaterales que estos producen.

E2. La electiva me permitió tener una visión más crítica acerca de lo que produce la ciencia y la tecnología; los científicos deben ser personas muy responsables que se interesen por no generar consecuencias graves en el medio ambiente.

E3. Me dio bases sólidas y me aportó nuevos conocimientos prácticos y muy útiles para actuar y crear conciencia en las personas que voy a formar.

E4. Hizo pensar que hago parte de una comunidad la cual está en constantes cambios. Aprendí lo importante que es pensar antes de actuar.

E5. Creo que las CS ayudan a formar en los profesionales la autonomía, la cual lleva a ser creativos y a decir lo que pensamos.

\section{Ventajas que ofrece la utilización de las CS}

Las CS permiten el trabajo en pequeños grupos, el aprendizaje cooperativo, las discusiones centradas en los estudiantes, la resolución de problemas, las simulaciones y los juegos de roles, la toma de decisiones, el debate. Potencializan además la responsabilidad, desarrollando en los estudiantes la comprensión de su papel como miembros de la sociedad, que les permite asumir diversos roles desde el campesino, el empresario, el político, el científico. De acuerdo a Sadler y Zeidler (2004), estos roles hacen que los estudiantes propongan distintas explicaciones y cuestionamientos, que les permiten preparase para enfrentar de manera correcta diversas problemáticas, además de integrarse en un conjunto más amplio que constituye su propia naturaleza, y contemplan las influencias mutuas entre la ciencia, la tecnología, la sociedad y el ambiente, promoviendo puntos de vista equilibrados para que los estudiantes tengan la posibilidad de elegir conociendo diversas opiniones.

Promociona una acción responsable, motivando a los estudiantes hacia visiones más amplias de la ciencia, la tecnología y la sociedad que incluyen gestiones éticas y de valores, lo que permite a los estudiantes tomar decisiones fundamentadas en opiniones colectivas, en valores y en reflexiones críticas, que no simplemente vayan de la mano del sentido común, sino que se requiere una reflexión y evaluación profunda de cada una de las acciones que acompañan el diario vivir.

Promueve la responsabilidad social, a lo cual Santos y Mortimer (2001) afirman que un sujeto se puede considerar agente responsable cuando asume un problema social como una preocupación personal. El estudiante comprende que cualquier tipo de decisión individual tiene implicaciones sociales, y es aquí donde se puede ver un mayor interés en una educación por actitudes y valores, lo cual requiere un compromiso serio por parte de todas las comunidades (ciudadanos, consumidores, profesionales) para enfrentar la crisis ambiental.

Las CS favorecen actitudes y valores como el consumo responsable; de manera que propician en los estudiantes reflexiones sobre la necesidad de comprar productos con garantía que han sido obtenidos con procedimientos sostenibles, respetuosos con el medio y con las personas (Vilches, Praia \& Gil-Pérez, 2008).

A continuación se presenta un fragmento de los debates que eran grabados y que hacían parte de la metodología utilizada; en la que se pueden identificar intervenciones de distintos actores sociales representados por los estudiantes como: Campesinos (C), empresarios (E) y gobernantes (G).

C. Soy representante de la comunidad de San Juan y estoy aquí para denunciar acerca de los graves daños a los que nosotros hemos sido víctimas como consecuencia de las fumigaciones del glifosato, hemos visto que últimamente las cosechas se han quemado, los animales están presentando daños físicos, además, de la aparición de las manchas en la piel e irritación en los ojos en mis vecinos. Mi pregunta va dirigida a los fabricantes del producto acerca si son conocedores de este mal y al gobierno si ha buscado formas de subsanar tales males.

E. De acuerdo con las recomendaciones de nuestra empresa esto [glifosato] no representa daño alguno, si se respetan las tasas de aplicación, para plantas leñosas como la coca, y de acuerdo a estudios por otras entidades se observa que la mezcla está clasificada como ligeramente tóxica; pero considerando la situación presentada por la comunidad, se puede 
indicar que los encargados de realizar las respectivas mezclas están utilizando cantidades mayores a las recomendadas, además, ustedes deben informarse acerca de los posibles efectos que tiene cualquier tipo de producto que se utilice.

C. Nosotros no somos los que lo utilizamos, nuestra comunidad estaba bien hasta cuando ustedes empezaron a utilizar este veneno, además nos gustaría saber si la información que ustedes acaban de dar, solo corresponde a un estudio o se han realizado estudios más seguidos a partir de la aplicación de dicho producto.

G. El plan de gobierno no puede permitir que se continúe con la implementación de cultivos ilícitos, por lo tanto, si la comunidad no quiere que se siga utilizando estos químicos debería también comprometerse a no sembrar la coca y a denunciar a las personas que cometen este delito, sin embargo, son pocas las denuncias que hasta el momento se han presentado pero dada la gravedad de la situación nos comprometemos a buscar otras alternativas de erradicación tales como la erradicación manual, la creación de concejos comunales y otras alternativas de economía.

C. Están acabando con los animales y las plantas que teníamos, no han medido las consecuencias que este químico ha traído a nuestros campos y cómo está afectando lo que nosotros queremos, que también debería interesarle a ustedes porque estamos en el mismo planeta.

Los anteriores fragmentos permitieron asumir distintos roles por los estudiantes, lo que favoreció procesos de educación dialógica desde distintas perspectivas, facilitando la posibilidades de participar crítica y responsablemente frente a otras controversias de la sociedad actual.

\section{Reflexiones que requieren hacer los docentes}

La atención a las relaciones CTSA ᄀ-CS, debe verse como una orientación de la enseñanza absolutamente necesaria para modificar la imagen deformada de la ciencia, la cual debe ser vista por los estudiantes como una construcción humana, con múltiples controversias, que requiere ser desarrollada por personas responsables y éticas, por lo que los docentes deben tener una convicción acerca de la necesidad de educar para la sostenibilidad, además de poseer capacidades para orientar discusiones en el aula de clase acerca de la naturaleza de la ciencia, los aspectos sociológicos, políticos, éticos y las implicaciones ambientales que estos avances han tenido.

El desempeño profesional de los docentes debe permitir no solo un desempeño personal, sino también social, animado por interacciones sociales, vivencias, experiencias, reflexiones y aprendizajes que pueden ocurrir en los diversos contextos, donde se van a desenvolver profesionalmente (Loucks-Horsley, Hewson, Love \& Stiles, 1998; Day, 1999; Ponte y Oliveira, 2002).

Feldman (2000), recomienda que los profesores propicien cambios transformativos si reconocen la inconsistencia de sus concepciones y están abiertos a una nueva orientación, que les permita abordar diversos procesos de manera razonable, comprensible y benéfica ante situaciones particulares; por lo que es necesario, ser conscientes de la gravedad y del carácter global de los problemas a los que se enfrenta la humanidad. Es fundamental que el docente considere que sus alumnos aprenden a partir de la experiencia directa, relacionada con la vida diaria (experiencia / vivencia), con la construcción de sus propios significados.

De acuerdo a Stenhouse (1970), el docente debe asumir un papel de dinamizador que formula preguntas, utiliza problemas, promueve reflexiones y mantiene un ritmo interesante en la discusiones de su práctica pedagógica, promueve la autocrítica a través de preguntas precisas; así, es posible formar personas críticas capaces de actuar con responsabilidad social, escenario indispensable para abordar las CS en el aula de clase.

\section{CONCLUSIONES}

Es conveniente la inclusión de acciones educativas que promuevan una educación para la ciudadanía, que permitan una participación social fortaleciendo acciones colectivas, oponiéndose a actitudes negativas encaminadas a la destrucción de la biodiversidad. Se deben promover acciones de solidaridad donde se defienda una cultura de convivencia. 
El abordaje de cuestiones sociocientíficas, permite estudiar aspectos de la vida cotidiana y permite procesos de enseñanza y aprendizaje desde las aplicaciones de nivel de competencia de los futuros profesionales, profundizando conceptos representativos con efectos en aspectos sociales, económicos, culturales. Promueve reflexiones sobre la finalidad de la enseñanza de una disciplina, desarrollando actitudes acerca del desempeño profesional individual y colectivo en la sociedad.

Es posible promover actitudes y valores de conservación y educación para la sostenibilidad, si hay interés de los docentes por incorporar las cuestiones sociocientíficas en los currículos, donde se establece una relación coherente entre los procesos de enseñabilidad y la cotidianidad.

\section{REFERENTES BIBLIOGRÁFICOS}

- Acevedo, J. A. (1995). Educación tecnológica desde una perspectiva CTS. Una breve revisión del tema. Alambique, 3, 75-84.

- (2006). Relevancia de los factores no-epistémicos en la percepción pública de los asuntos tecno científicos. Revista Eureka sobre Enseñanza y Divulgación de las Ciencias, 3(3), 370-391. En linea en: http://www.apaceureka.org/revista/Volumen3/Numero_3_3/Acevedo_2006.pdf. Consultado 20 Septiembre de 2010.

- Aikenhead, G. S. (1997). Toward a First Nations Cross-Cultural Science and Technology Curriculum. Science Educación, 81(2), 217-238.

- Colucci-Gray, L., Camino, E., Barbiero, G., y Gray, D. (2006). From Scientific Literacy to Sustainability Literacy: An Ecological Framework for Education. Science Education, 90, 227252.

- Comín, P., y Font, B. (1999). Consumo sostenible. Barcelona: Icaria.

- Day, C. (1999). Developing teachers: The challenges of lifelong learning. London: The Falmer Press.

- Feldman, A. (2000). Decision making in the practical domain: A model of practical conceptual change. Science Education, 84, 606-623.

- Lederman, N. G. (1999). Teachers' understanding of the nature of science and classroom practice: Factors that facilitate or impede the relationship. Journal of Research in Science Teaching, 36(8), 916-929.

- Loucks-Horsley, S., Hewson, P., Love, N., y Stiles, K. (1998). Designing professional development for teachers of science and mathematics. Thousand Oaks, CA: Corwin Press.

- McComas, W. F., y J. K. Olson. (1998). The nature of science in international standards documents. En W. F. McComas, (Ed.), The Nature of Science in Science Education: Rationales and Strategies. (pp. 41-52). Dordrecht, The Netherlands: Kluwer Academic Publishers.

- Ponte, J. P., y Oliveira, H. (2002). Remar contra a maré: A construção do conhecimento e da identidade profissional na formação inicial. Revista de Educação, 11(2), 145-163.

- Reis, P. (2001). O ensino das ciências através da discussão de controvérsias: realidade ou ficção? En B. D. Silva \& L. S. Almeida, (Org.), Actas do VI Congresso Galaico-Português de Psicopedagogia. (pp. 367-379). Braga: Centro de Estudos em Educação e Psicologia da Universidade do Minho.

- Rubba, P. A., y Harkness, W. L. (1993). Examination of preservice and in-service secondary science teachers' beliefs about Science-Technology-Society interactions. Science Education, 77(4), 407-431.

- Rubba, P. A., Schoneweg-Bradford, C. S., y Harkness, W. J. (1996). A new scoring procedure for the Views on Science-Technology-Society instrument. International Journal of Science Education, 18(4), 387-400.

- Sadler, T. D., y Zeidler, D. L. (2004). The Morality of Socioscientific Issues: Construal and Resolution of Genetic Engineering Dilemmas. Science Education, 88, 4-27.

- Santos, W., y Mortimer, E. (2001). Tomada de decisão para ação social responsável no ensino de ciências. Ciência \& Educação, 7(1), 95-111.

- Stenhouse, L. (1970). The Humanities Project: an introduction. London: Heinemann.

- Vilches, A., y Gil-Pérez, D. (2003). Construyamos un futuro sostenible. Diálogos de supervivencia. Madrid: Cambridge University Press.

- Vilches, A., Praia, J., y Gil-Pérez, D. (2008). O Antropoceno: Entre o risco e a oportunidade, Educação. Temas e Problemas, 5, Año 3, 41-66.

- Wang, H., y Schmidt, W. (2001). History, philosophy and sociology of science in science education: results from the third international mathematics and science study. Science \& Education, Netherlands, 10, 51-70 
1. Docente Facultad de Ciencias de la Educación, Universidad Pedagógica y Tecnológica de Colombia. 